\title{
Comparative study between the Er:YAG and Nd:YAG lasers in treatment of post acne scar
}

\author{
Sameh M El-Taher, MD; Soha F El-Mekkawy, MD; Fathy Khodier, MD
}

a) Department of Plastic and Reconstructive Surgery, Ain Shams University, Cairo, Egypt.

b) Department of Plastic and Reconstructive Surgery, Cairo University, Cairo, Egypt.

\begin{abstract}
Background: Acne vulgaris is a very common skin disorder that affects virtually all individuals at least once during life. Acne scarring can have a great impact on the psychosocial life for the young adults. Er:YAG lasers has been used in the treatment of acne scarring. The results, however, produced open wounds with significant downtime. In contrast, nonablative remodeling lasers, (1064-nm Nd:YAG lasers) can reduce acne scarring without significant downtime, but at lower efficacies. Recently, resurfacing with fractional microscopic delivery of high energies to targeted depths in the dermis has made possible significant clinical improvements often approaching that of ablative lasers without any reports of permanent hypo pigmentation or scarring. So the aim of this study was to determine the best modality in treatment of acne scars.

Patients and methods: 26 patients with mild to moderate facial acne scarring were included in this study, they were divided into 3 groups using traditional ablative Er:YAG laser, Nd:YAG laser and fractional Er:YAG laser respectively. Histopathological specimen were taken for objective assessment, and subjectively by patient satisfaction, doctor satisfaction.

Results: Showed that the use of fractional Er:YAG laser had the greatest clinical improvements in scar treatment, with mild erythema without any downtime. With edema not lasting for more than 1 day. No crusting, scabbing formation, or infection was seen in this group.

Conclusion: Er:YAG laser fractional photocoagulation is a promising option for the treatment of atrophic post acne scars, avoiding the adverse effects of ablative laser procedures with improving the limited efficacy of the nonablative ones.
\end{abstract}

\section{Introduction:}

Acne vulgaris is one of the most prevalent and potentially physically and emotionally debilitating dermatologic diseases that affect nearly $80 \%$ of adolescents and young adults aged 11 to 30 years. Lesions appear mainly on the face, back, chest, and other areas where a great number of pilosebaceous glands exist. Facial acne scarring affects approximately $95 \%$ of acne patients and is related to excessive inflammation, initial acne severity, and the time passed before seeking adequate treatment. ${ }^{1}$

The inflammatory stage of acne causes permanent skin changes and fibrosis. Scars normally pass through the normal stages of the wound-healing sequence: inflammation, granulation, and remodeling. Dermal damage is more long lasting and results in an increase or decrease of tissue and had bad look with age as a result of normal skin changes. Actually, damage to the epidermis or papillary dermis only will heal without any scar formation. ${ }^{2}$

Scarring is most commonly atrophic; Jacob et $\mathrm{al}^{3}$ classified acne scarring into 3 main types: icepick, rolling, and boxcar. The icepick scars are usually smaller in diameter $(<2 \mathrm{~mm})$ and deep which extends to the dermis or subcutaneous tissue. The opening is smaller and steep-sided; there may be a wide base that could evolve into a depression. Boxcar scars were described as shallow $(<0.5 \mathrm{~mm})$ or deep (> $0.5 \mathrm{~mm}$ ) and are often 1.5 to $4 \mathrm{~mm}$ in diameter. They have well defined edges with steep, almost vertical walls. Soft rolling scars 
have different shapes; circular or linear, which are often larger than $4 \mathrm{~mm}$ in diameter. They have gently sloped edges which continue with normal skin. There may be accompanied with dermal or subdermal wrinkle. It may show hypertrophy or show keloid. Hyper pigmentation occurs more commonly in patients with darker colors. Both scarring and hyper pigmentation may result in bad disfigurement. $^{3}$

Various therapeutic measures have been employed to reduce acne scarring, such as punch excision, punch elevation, dermabrasion, subcutaneous incision (Subcision), chemical peeling, and the injection of fillers, but most of these modalities are associated with suboptimal results. Dermabrasion and chemical peel had been the mainstay therapeutic modalities for treating acne scarring for a long period of time. Dermabrasion involved surgical removal of the skin with either a wire brush or a diamond fraise. There was clear variability in the ability to exfoliate tissue in a uniform and accurate way. This requires years of experience and technical skill to obtain good results. ${ }^{4}$

On the other hand, Chemical peeling was based on scientific principles; skin types, morphology and preparation of patient skin to obtain a good results. The ideal modality of peeling was to provide extremely precise, uniform, and controlled tissue exfoliation with minimal complications. ${ }^{4}$

The theory of selective photothermolysis made laser treatments significantly safer by allowing for selective irradiation of specific target chromophores with minimizing damage to adjacent structures..$^{5}$ Ablative lasers such as the carbon dioxide (CO2) and erbium:yttriumaluminum-garnet (Er:YAG) laser, remain the gold standard in skin resurfacing and provide the greatest clinical improvements with the least number of sessions. So, because of the total vaporization of the epidermis and different coagulative damage to the dermis, healing time is longer and treatment shows increased incidence of complications. On the other hand, only superficial resurfacing could be done because of the fear of permanent hypopigmentation and scarring if deeper resurfacing is done. 6
In contrast, nonablative lasers such as the 1320-nm or 1064-nm neodymium:yttriumaluminum-garnet lasers (Nd:YAG), diode $(1450 \mathrm{~nm})$ and the $585-\mathrm{nm}$ pulsed dye laser (PDL), minimize these risks by leaving the epidermis intact while delivering dermal laser irradiation with associated surface cooling measures. The avoidance of epidermal damage appears to lack the mechanism of a true wound healing process and therefore decreased resurfacing efficacy. Multiple treatment sessions were required and there are variable clinical improvements in scar appearances, $40 \%$ to $50 \%$ had been observed following treatments. ${ }^{7}$

Fractional resurfacing is a novel variation on the theory of selective photothermolysis wherein microscopic treatment zones (MTZs) of controlled width, depth, and densities are created. These controlled zones of thermal heating and tissue damage were surrounded by spared areas of viable epidermis and dermis that allow for rapid repair of the microscopic treatment zones. ${ }^{8}$

After laser session, visible columns of both epidermal and dermal area of thermal damage were seen with intact skin coverage. Within 24 hours, there is migration of viable cells from the periphery of the microscopic treatment zones (MTZs) with formation of microscopic epidermal necrotic debris (MENDs) ${ }^{9}$ that undergo transepidermal extrusion between 3 and 7 days with clinical improvements in scar appearances of $50 \%$ to $70 \%$ had been observed following treatments. ${ }^{10}$

Our purpose of this study is to provide a safe and comprehensive approach to the best modality for the management of post acne scarring.

\section{Patients and methods:}

This study was performed between March 2008 to March 2009 on 26 patients (7 males, 19 females). Written consent was taken from patients before the treatment sessions. Patients were photographed as follows; before starting the laser session, after each session and 3months post laser treatment using identical camera settings and lighting conditions. Inclusion criteria were; age (21 - 45 years) with fitzpatrick skin type III and IV, ${ }^{11}$ only mild to moderate 
acne scarring were included in this study. Exclusion criteria were; known photosensitivity, pregnancy or lactation, the use of isotretinoin, a history of previous facial laser treatment or facial surgical procedure within last 6 months, severe acne scars due to combination of different modalities in treatment of such cases, patients with medical conditions that might interfere with proper wound healing e.g. diabetes mellitus, patients on corticosteroid therapy ... etc.

The patients were divided into three groups (The device used was Dualis SP II);

Group A; we used traditional Er:YAG laser on 9 patients for 1 session only done with 4 $\mathrm{mm}$ spot size were used with fluence $2 \mathrm{~J} / \mathrm{cm} 2$ and repetition rates $5 \mathrm{~Hz}$, Group B; we used $\mathrm{Nd}$ :YAG laser $(1064 \mathrm{~nm})$ on 8 patients for 7 sessions 3 weeks apart at a fluence of $70 \mathrm{~J} / \mathrm{cm} 2$ and a $50 \mathrm{~ms}$ pulse duration using a $7-\mathrm{mm}$ spot size. Two passes of laser were made in each session and associated air cooling was used to decrease the epidermal temperature. Group C; we used Fractional Er:YAg laser on 9 patients for 3 sessions 4 weeks apart, in each session we used 4 passes at 400 microseconds pulse duration $7 \mathrm{~mm}$ spot size with 1 joule per pulse.

Histopathological assessment was done using 3 biopsies (2-mm punch biopsy) as follows; control biopsy was taken from the normal facial skin (i.e. pre auricular area), another one from untreated scars before treatment, and final one 3 months after finishing the treatment (biopsies are not taken from the previous same area). Biopsy specimens were fixed in $10 \%$ buffered formalin and embedded in paraffin sections, and then they were stained with Hematoxylin-Eosin and Masson Trichrome.

Acne scars improvement was assessed by patient satisfaction, doctor satisfaction and by analysis of histopathological assessment with photographs of the patients taken pre and post to the laser sessions.

\section{Results:}

26 patients (7 male, 19 female) were included in this study. Ages ranged from 21 to 45 years (average 23) and Fitzpatrick skin types were III and IV.

\section{Group A: Clinical Evaluation:}

Data revealed that the use of traditional Er:YAG laser in all patients showed pain that lasted for 2 days (average 1-4 days) followed by discomfort for another 3 days (average 28 days) that was controlled by oral analgesics; intramuscular analgesia was given in 5 patients $(55.5 \%)$ immediately post laser. Hyperemia and redness extended for 7 days (average 5 11 days) in 3 patients (33.3\%), in 2 cases $(22.2 \%)$ the redness extended for more than 3 weeks and changed into hyperpigmentation which was treated by local bleaching creams and improved within 3 months (no cases had hypo pigmentation). Edema lasted for 3 days (average 2 - 7 days) that gradually subsided. Only in 1 case $(11.1 \%)$ the edema lasted more than 10 days and the patient was given corticosteroid injection once and oral antihistaminic. Crusting or scabbing formation was seen in these patients which resolved after 4 days (average 2 - 12 days). Infection was seen in only one patient $(11.1 \%)$ which resolved after 6 days on topical and oral antibiotics. After healing, there was improvement of the previous post acne scars. New scars were seen in 2 cases $(22.2 \%)$ especially at the sites of older deep scars. The presence of edges between the normal skin and scarred one was still found in 2 cases (22.2\%). Care was given during resurfacing to avoid their appearance. 


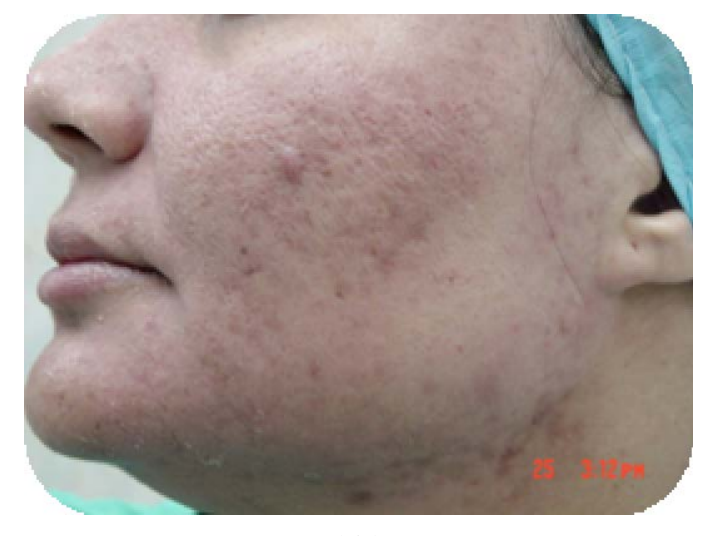

(A)

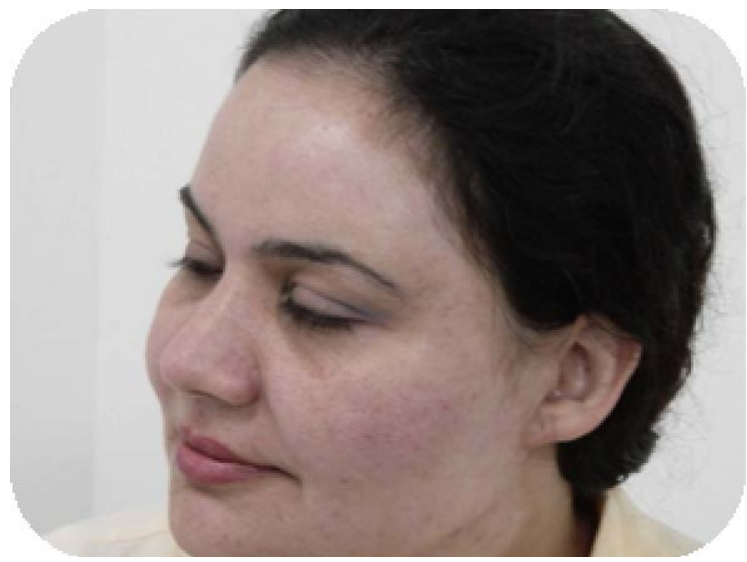

(C)

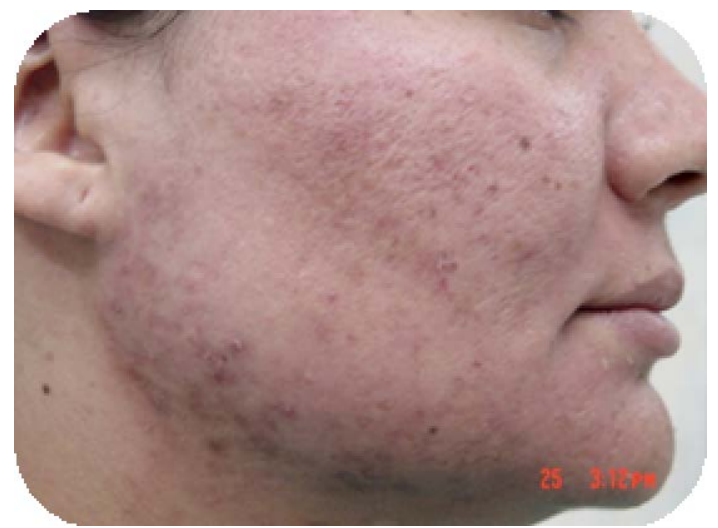

(B)

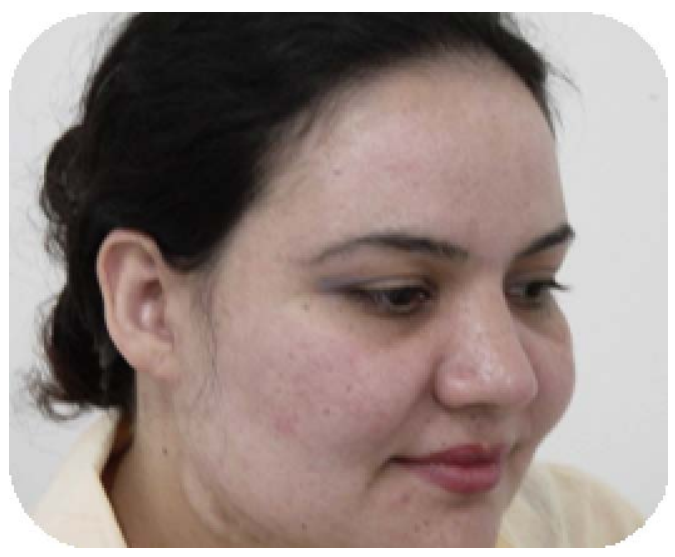

(D)

Figure (1): (A,B) Showing a patient with post acne scarring, $(C, D)$ shows the results after laser resurfacing using traditional Er:YAG laser.

\section{Histopathologic evaluation:}

Revealed epidermal thinning (only basal layer of epidermis was found in some areas), scar tissue organization and increased collagen and fibrous tissue deposition, increased fibroblasts and macrophages. Collagen deposition was significantly increased, as evaluated using Hematoxylin-Eosin and Masson Trichrome.

\section{In Group B: Clinical Evaluation:}

$\mathrm{Nd}: Y A G$ laser was used in all patients. As regard the pain it lasted for 2 hours (average 0-12 hours), no discomfort was seen and there was no need for oral analgesics except in 1 patient (12.5\%) who took it for 24 hours only. Hyperemia and redness did not extend more than 12 hours (average 6-24 hours) in 2 patients $(25 \%)$ and the redness didn't last for more than 3 days with no changes in the color of the treated area (no hyper/ hypo pigmentation). Edema occurred only in 1 case $(12.5 \%)$ that lasted for only 1 day then gradually subsided by ice packs. No crusting, scabbing formation or infection was seen in this group. At the end of the treatment ( 7 sessions) there was considerable improvement of the previous post acne scars.
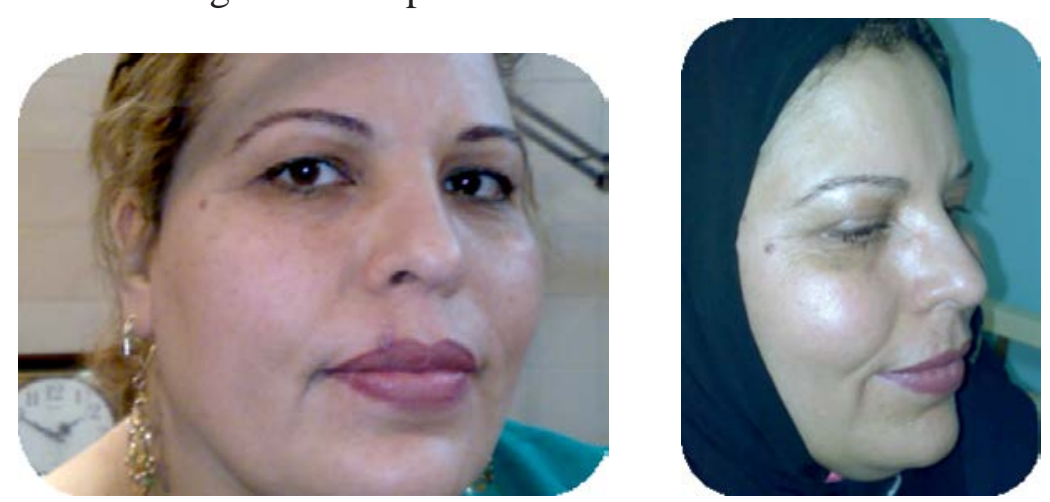

Figure (2): Showing a patient treated with non ablative Nd:YAG laser. 
Histopathologic evaluations showed mild epidermal thinning; scar tissue reorganization and increased collagen deposition with minimal fibrous tissue deposition observed with the new collagen network. In particular, collagen deposition was significantly increased after all treatments.

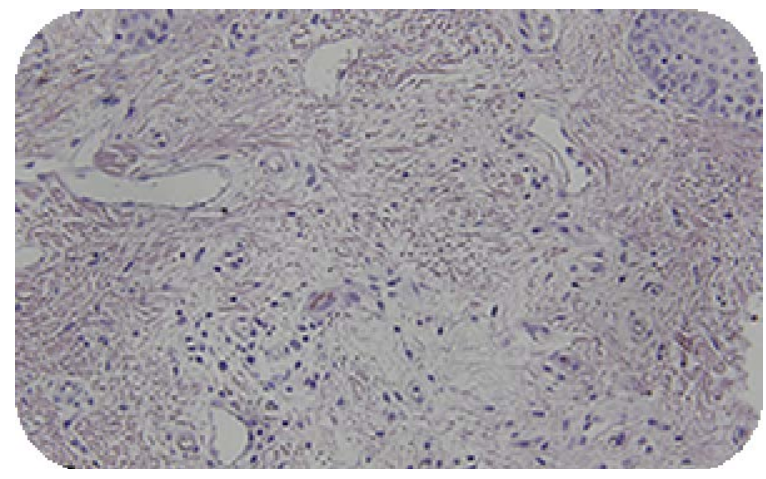

(A)

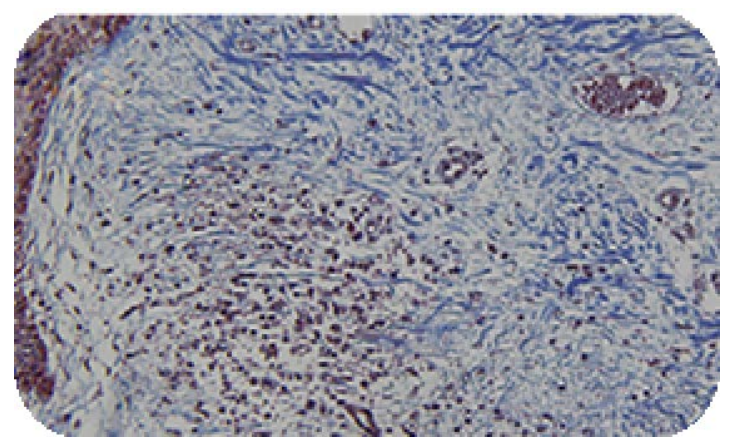

(C)

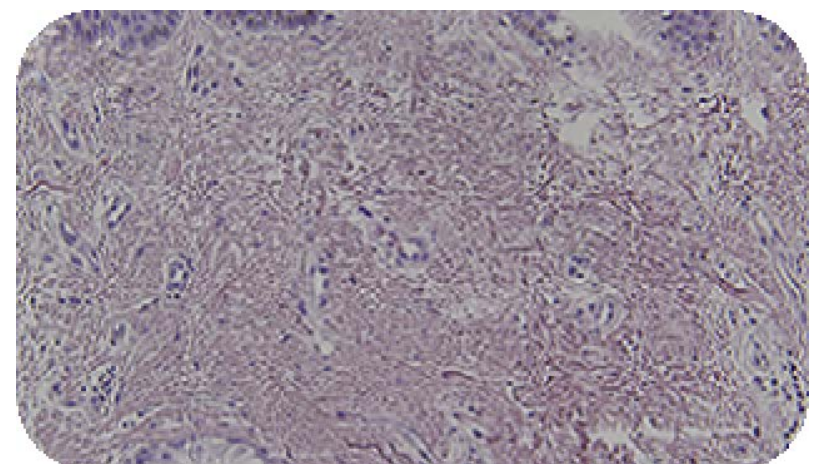

(B)

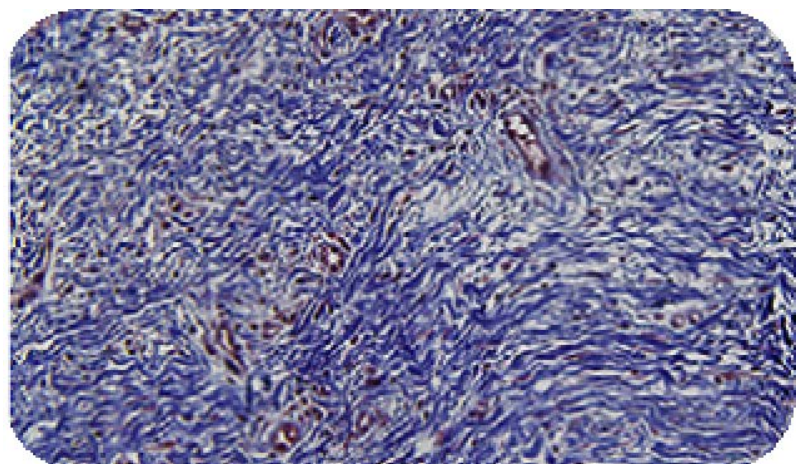

(D)

Figure (3): Histologic evaluations of atrophic acne scars revealed increased collagen deposition after completing laser treatment with fractional Er:YAG laser as stained by Hematoxylin-eosin stain A) pre laser, B) post laser and Masson-trichrome stain, C) pre laser, D) post laser.

Group C: the use of fractional Er:YAG laser (high energy with deep tissue coagulation) showed greatest clinical improvements in scar treatment. As regard the pain it lasted for 6 hours (average 2-18 hours), mild discomfort was seen and resolved within the 1 st 24 hours, only one patient $(11.1 \%)$ developed post inflammatory hyperpigmenation, another one case $(11.1 \%)$ showed localized scarring that

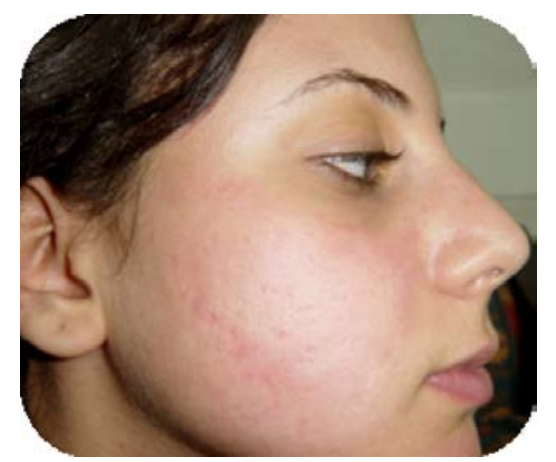

resolved with intralesional corticosteroid injection. The erythema lasted for 1 day (average 0-4 days); in 1 case $(11.1 \%)$ it lasted more than 5 days and resolved by topical antibiotic ointement. In this type of treatment, erythema was mild without any downtime. Edema didn't last for more than 1 day. No crusting, scabbing formation, or infection was seen in this group.

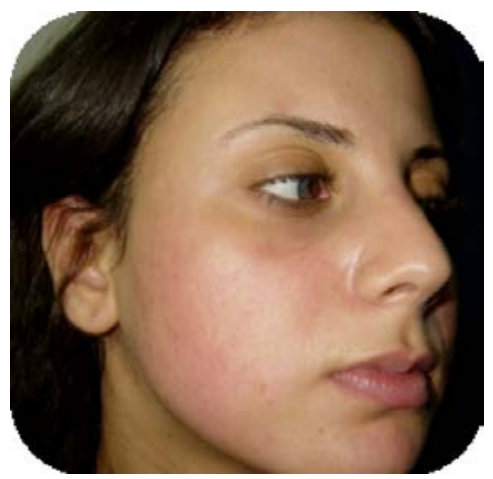

Figure (4): Showing a patient treated with fractional Er:YAG laser. 
Histopathologic evaluation showed epidermal and dermal thermal injuries with formation of microscopic epidermal necrotic debris (MENDs) with normal stratum corneum of epidermis (biopsy taken during treatment). At the end of treatment the scar tissue showed organization and marked increase in collagen deposition with minimal fibrous tissue deposition. There was increase in elastin with minimal fibroblasts and macrophages.

\section{Discussion:}

Atrophic acne scarring occurs due to impaired resolution or healing of damage caused in and around pilosebaceous glands during active inflammation. These scars are usually classified according to shape and depth as ice-pick, rolling, and boxcar scars; they involve deeper structures and draw in surface layers to cause indention or atrophy. In addition, tissues are also destroyed by enzymatic activities and by the actions of inflammatory mediators released from disrupted acne follicles and cysts which may aggrevate the condition. Laser skin resurfacing has revolutionized the treatment of atrophic acne scars; they still present a major therapeutic challenge. ${ }^{12}$

Ablative resurfacing with the Er:YAG laser remains the standard method in treatment of atrophic post acne scars and skin rejuvenation. It is associated with considerable healing time with the risk of prolonged redness, erythema, edema, infection, scabbing and scarring. Er:YAG laser is a painful procedure and usually requires general anesthesia in the operating theatre. ${ }^{1,13}$ It has an efficacy of $25 \%$ to $90 \%$ for treating acne scars. Pigmentary alterations are common complications, especially postoperative hyperpigmentations but it is usually transient and it is common in patients with darker skin types. There is increase in the amount of collagen deposition and elastin in dermal layer in patients who are treated with Er:YAG laser. ${ }^{14}$

In this study pain, discomfort, edema and infection were not common. They occurred in the mild form and healed spontaneously with minimal intervention. The only complication that occurred and needed long follow up was hyperpigmentation $(22.2 \%)$. It lasted more than 3 months and there was mild improvement.
There was formation of new collagen with increase of its deposition in the dermal layer of skin. The overall improvement ranges from $30 \%-70 \%$ in treating post acne atrophic scars.

Due to the hazards and disadvantages of ablative laser therapy, researchers turned to nonablative lasers. At first they started with intense pulsed light lasers. These were found to be safe but limited in efficacy, and the results were not comparable to ablative resurfacing. Then they shift to use local resurfacing with a $1064 \mathrm{~nm} \mathrm{Nd:YAG} \mathrm{nonablative} \mathrm{laser} \mathrm{using}$ an array of microscopic thermal wounds. It proved effective, with reasonable healing time and morbidity was minimal. However, the procedure required multiple sessions (6-8 sessions) with local anesthesia, and the results were sometimes variable. ${ }^{7,15}$ On the other hand, nonablative lasers (the 1064-nm Nd:YAG laser) are known to change acne scar appearance by stimulating collagen production and dermal remodeling. The collagen fibers in the papillary dermis are increased in density and number. The elevation occurred at 8 weeks after treatment completion, and this corresponded to clinical improvements observed by physicians and patients. Nd:YAG laser was most effective in treating superficial boxcar and rolling scars and was less effective in treating deep boxcar, deep rolling, and icepick scars. There is improvement in scar appearances (40\%-50\%) following the nonablative laser treatments with minimal side effect and lower efficacy than Er:YAG ablative laser. $^{7}$

In this study, Nd:YAG laser for the treatment of acne scars has showed mild improvements for all types of atrophic acne scars with minimal healing time, minimal pain, edema, erythema, crust and scars. There was no infection, no hyper or hypo pigmentation which was commonly seen in ablative laser. Nd:YAG was more effective in treating the superficial acne scars more than deeper one. There was mild epidermal thinning, scar tissue reorganization and increased collagen deposition with minimal fibrosis. The overall improvement ranged from 20\%-50\% in treating post acne atrophic scar after 7 sessions of treatment.

Manstein et al, $2004^{16}$ introduced the concept of fractional photothermolysis to bridge 
the gap between ablative and nonablative resurfacing. A novel modification of the fractional photothermolysis technique, from "bulk ablation" to "localized ablation". The laser produces thousands of microscopic, clinically inapparent thermal wounds in the skin, while the intact undamaged skin around each wound acts as a reservoir, allowing relatively rapid reepithelialization of the treatment zone with little risk of infection and scarring. ${ }^{16}$

Fractional delivery systems for Er:YAG lasers developed to achieve the clinical results observed with traditional ablative lasers. These devices cause true ablation of the epidermis in addition to variable depths of ablative damage to the dermis. ${ }^{17}$ The combination of epidermal and dermal ablation increased the wound healing response and accompanying dermal fibrosis, which may explain the rapid and significant clinical effects that can be achieved with ablative versus nonablative devices. Fractional ablation lead to microscopic ablation of scar tissue, normal skin is preserved in adjacent areas, and healing time as well as scarring risk is minimized compared with conventional ablative lasers. ${ }^{18}$ Histopathological studies revealed discrete microthermal zones of epidermal and partialthickness dermal coagulation that increase in both depth and width with increasing pulse energies. The significant depth of penetration in the fractional lasers leads to improvements in scars similar to those treated with the ablative lasers. The great advantage of fractional lasers is that it can be safely used on all skin types without risk of pigmentary alteration. ${ }^{19}$

In our study, fractional ablative lasers were changed effectively more than nonablative procedures, with predictable results. Pain, discomfort, edema and infection were not detected. Only redness and erythema were occurring for little extent. Hyperpigmentation which is the main disadvantage of ablative laser resurfacing was not noted in our patients. This may be due to the microscopic pattern of injury induced by the Er:YAG laser which caused only minimal inflammation and led to fewer clinically evident pigmentary changes (hyperpigmentation is a long-term sequel of any type of laser treatment, and it needed long follow-up before it can be completely resolved).
The biopsy samples showed the epidermal and upper dermal ablation and healing process with collagen regrowth. The overall improvement ranged from $50 \%-70 \%$ in treating post acne atrophic scar with no permanent side effects. Further comparative studies of the use of fractional lasers are needed to confirm the efficacy and safety of this technique.

In conclusion, Er:YAG laser fractional photothermolysis is a promising option for the treatment of various dermatologic conditions (especially atrophic post acne scars), avoiding the adverse effects of ablative laser procedures with improving the limited efficacy of the nonablative ones.

\section{References:}

1- Jordan R, Cummins C, Burls A: Laser resurfacing of the skin for the improvement of facial acne scarring: a systematic review of the evidence. Br J Dermatol 2000; 142: 413-423.

2- Del Rosso JQ: Acne in the adolescent patient: interrelationship of psychological impact and therapeutic options. Today's Their Trends 2001; 19: 473-484.

3- Jacob CI, Dover JS, Kaminer MS: Acne scarring: a classification system and review of treatment options. J Am Acad Dermatol 2001; 45: 109-117.

4- Lee HS, Lee JH, Ahn GY, Lee DH, Shin JW, Kim DH, et al: Fractional photothermolysis for the treatment of acne scars: a report of 27 Korean patients. $J$ Dermatol Treat 2008; 19: 45-49.

5- Anderson RR, Parrish JA: Selective photothermolysis: Precise microsurgery by selective absorption of pulsed radiation. Science 1983; 220: 524-527.

6- Trelles MA: Laser ablative resurfacing for photorejuvenation based on more than a decade's experience and 1200 patients: Personal observations. J Cosmet Dermatol 2000; 2: 2-13.

7- Lee DH, Choi YS, Min SUK, Yoon MY, Suh SDH: Comparison of a 585-nm pulsed dye laser and a 1064-nm Nd:YAG laser for the treatment of acne scars: A randomized split-face clinical study. Journal of American Academy of Dermatology 2009; 60(5). 
8- Laubach H, Chan HH, Rius F, et al: Effects of skin temperature on lesion size in fractional photothermolysis. Lasers Surg Med 2007; 39: 14-18.

9- Dierickx C, Khatari K, Altshulder G, et al: Fractionated delivery of Er:YAG laser light to improve the efficacy and safety of ablative resurfacing procedure. Lasers Surg Med 2007; 19: 16 (Abstract).

10-Taub AF: Fractionated delivery systems for difficult to treat clinical applications: Acne scarring, melasma, atrophic scarring, striae distensae, and deep rhytides. $J$ Drugs Dermatol 2009; 6(11): 1120-1128.

11-Fitzpatrick RE, Rostan EF, Marchell N: Collagen tightening by carbon dioxide versus erbium:YAG laser. Lasers Surg Med 2000; 27: 395-403.

12-Goodman GJ: Management of post-acne scarring: What are the options for treatment? Am J Clin Dermatol 2000; 1: 3-17.

13-McDaniel DH, Ash K, Lord J, Newman J, Zukowski M: The Erbium: YAG Laser: A review and preliminary report on resurfacing of the face neck and hands. Aesthetic Surgery Journal 1997; 17(3): 157 -164 .
14-Kaufmann R, Beier C: Erbium:YAG laser therapy of skin lesions; Med Laser Appl 2001; 16: 252-263.

15-Tanzi EL, Alster TS: Comparison of a 1450nm diode laser and a 1320-nm Nd:YAG laser in the treatment of atrophic facial scars: A prospective clinical and histologic study. Dermatol Surg 2004; 30: 152-157.

16-Manstein D, Herron GS, Sink RK, Tanner $\mathrm{H}$, Anderson RR: Fractional photothermolysis: A new concept for cutaneous remodeling using microscopic patterns of thermal injury. Lasers Surg Med 2004; 34(5): 426-438.

17-Wanner M, Tanzi E, Alster TS: Fractional photothermolysis: Treatment of facial and nonfacial cutaneous phtodamage with a 1,550-nm erbium-doped fiber laser. Dematol Surg 2007; 33: 23-28.

18-Khatri KA, Machado A, Magro C, Davenport S: Laser peel: Facial rejuvenation with a superficial erbium:YAG laser treatment. J Cutan Laser Ther 2000; 2: $119-123$.

19-Laubach HJ, Tannous Z, Anderson RR, et al: Skin responses to fractional photothermolysis. Lasers Surg Med 2006; 38: 142-149. 